\title{
REDUCED BASIS MULTISCALE FINITE ELEMENT METHODS FOR ELLIPTIC PROBLEMS
}

\begin{abstract}
JAN S. HESTHAVEN *, SHUN ZHANG ${ }^{\dagger}$, AND XUEYU ZHU $\ddagger$
Abstract. In this paper, we propose reduced basis multiscale finite element methods (RB-MsFEM) for elliptic problems with highly oscillating coefficients. The method is based on multiscale finite element methods with local test functions that encode the oscillatory behavior ([4, 14]). For uniform rectangular meshes, the local oscillating test functions are represented by a reduced basis method, parameterizing the center of the elements. For triangular elements, we introduce a slightly different approach. By exploring over-sampling of the oscillating test functions, initially introduced to recover a better approximations of the global harmonic coordinate map, we first build the reduced basis on uniform rectangular elements containing the original triangular elements and then restrict the oscillating test function to the triangular elements. These techniques are also generalized to the case where the coefficients dependent on additional independent parameters. The analysis of the proposed methods is supported by various numerical results, obtained on regular and unstructured grids.
\end{abstract}

Key words. Multiscale finite element methods, reduced basis methods.

AMS subject classifications. TBD

1. Introduction. The development of efficient and accurate numerical methods for solving problems with highly oscillating coefficients is an area of research that is increasingly active. This is not only driven by applications in subsurface flows or the modeling of novel materials, but also by the prohibitive cost of solving such problems using a straightforward approach where all scales are adequately resolved.

To address and, ultimately overcome, the computational cost of resolving the finest scale, multiscale finite element methods (MsFEM) have been developed in [15, 16, 10, 17, 9]. In this approach, accuracy is achieved by locally solving a fine scale problem. These solutions are subsequently used to build the multiscale finite element basis, encoding the local fine structure, to capture the fine scale information of the leading order differential operator. Originally, MsFEM was proposed for linear finite elements but generalized in [4] to enable the use of high-order elements by local oscillating test functions (or harmonic coordinates). In [14, we proposed an alternative formulation of high-order MsFEM using related ideas, albeit introducing a more natural formulation.

The local oscillating test functions must be solved for each element of the coarse mesh and each of these local problems must be resolve to fully capture the local fine scale. Hence, these local problems has many degrees of freedom (DOFs) and a fast solver of these local problems is central to the efficiency of MsFEM. To address this, we consider the reduced basis method (RBM) as an ideal technique that provides an efficient representation of the solution to parametric problems in manyquery and real-time scenarios, see introductions in [23, 24, 25]. This idea has been pursued by a few authors recently in the context of multi-scale problems, as in [7, where reduced basis methods are used to represent the homogenized coefficients. In [21, the author use a set of reduced bases to represent multiscale finite element basis functions for parameter dependent problems using MsFEM developed previously in [16]. In [11, a reduced basis based method is discussed for MsFEM with

\footnotetext{
${ }^{*}$ Chair of Computational Mathematics and Simulation Science (MCSS), Mathematics Institute of Computational Science and Engineering (MATHICSE), Ecole Polytechnique Federale de Lausanne (EPFL), CH-1015 Lausanne, Switzerland, and Division of Applied Mathematics, Brown University, Providence, RI 02912, Jan.Hesthaven@epfl.ch.

${ }^{\dagger}$ Department of Mathematics, City University of Hong Kong, Kowloon Tong, Hong Kong, China, shun.zhang@cityu.edu.hk.

${ }^{\ddagger}$ Scientific Computing and Imaging Institute, University of Utah, Salt Lake City, UT, 84112, xzhu@sci.utah.edu.
} 
local eigenfunction basis functions and a local reduced basis discontinuous Galerkin approach is proposed for a two-phase flow problem in [19. In the context of the related FE-HMM, reduced basis finite element heterogeneous multiscale method is proposed in [1] and an adaptive version is discussed in 2].

In this paper, we focus on the development of reduced basis multiscale finite element method (RB-MsFEM) for MsFEM based on local oscillating test functions([4, 14]). The central element of the reduced basis method is the parametrization of the problems to be solved. For local oscillating test functions, it is natural to choose the locations of the elements as parameters. A tempting alternative is to choose all vertices as parameters. However, if local elements of different sizes and shapes have very different coefficients, even in the simplest case of periodic coefficients case, as a result, the resulting reduced basis set will be large and the method will loses its efficiency. A natural way to control the size of the reduced basis set is by working on meshes with uniform rectangular elements. This ensures that all elements have similar distributions of coefficients and we can expect the size of the reduced basis set to be reasonable.

However, purely rectangular meshes are clearly insufficient for many applications, presenting a contrast to the desirable geometrical flexibility of finite element methods. To extend RB-MsFEM to arbitrary triangular meshes, we shall explore the idea of oversampling. It is indeed well known that computing the local oscillating test functions in a larger domain results in an improved approximation to the globally defined oscillating test functions (harmonic coordinates) ([10, 17] for discussions about oversampling technique and [22] for a discussion of the globally defined oscillating test functions (harmonic coordinates)). In this work, we first build the reduced basis set on uniform rectangular domains containing the original triangular elements and then restrict the oscillating test function to the triangular elements. This approach is not completely independent of the DOFs on the local finite elements, but the most time-consuming part of the computation, solving the local finite element problem for the oscillating test functions, is done efficiently by a reduced basis method. We will generalize this approach to problems with coefficients depending on other independent parameters. It is also worth emphasizing that the ideas introduced in this paper can be generalized to other constructions of multiscale finite element methods.

The rest of the paper is organized as follows. The formulations of the model elliptic problem and some general theory of homogenization are discussed in Sec. 2. Multiscale finite element methods, based on local oscillating test functions are introduced in Sec 3. In Sec. 4, we introduce the RB-MsFEM for uniform rectangular meshes and generalizations to RB-MsFEM for triangular meshes are discussed in Sec. 5. In Sec. 6, RB-MsFEM for more general parameter dependent PDEs is discussed,and supporting numerical experiments are provided in Secs 4-6. We conclude with a few final remarks in Sec. 7 .

2. Model Problem. In the following we shall define the problem of interest and offer a few general results that will be useful later.

2.1. Elliptic PDEs with multiple scales. Let $\Omega \in \mathbb{R}^{\mathrm{d}}, d=1,2,3$ be an open polygonal/polyhedral domain. We consider the elliptic problem

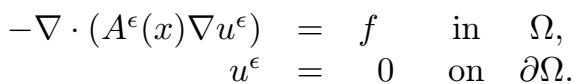

Here, the matrix $A^{\epsilon} \in L^{\infty}\left(\Omega, \mathcal{M}_{\alpha, \beta}\right)$, and $\mathcal{M}_{\alpha, \gamma}$ is a set of uniformly positive definite matrices with uniformly positive definite inverse, i.e., for any $\xi \in \mathbb{R}^{\mathrm{d}},\|\xi\|_{\ell^{2}}=1,0<\alpha \leq \xi^{T} A^{\epsilon} \xi \leq \beta^{-1}$. While the general homogenization theory is true for non-symmetric matrices, we restrict the discussion to the symmetric version for simplicity of the presentation. $A^{\epsilon}$ is allowed to vary on a fine scale $\epsilon$.

The variational formulation of the problem is to seek $u^{\epsilon} \in H_{0}^{1}(\Omega)$ such that

$$
a\left(u^{\epsilon}, v\right)=f(v) \quad \forall v \in H_{0}^{1}(\Omega),
$$


where

$$
a(u, v)=\left(A^{\epsilon}(x) \nabla u, \nabla v\right), \quad f(v)=(f, v) .
$$

2.2. General homogenization results. In the following, we summarize a few important results from homogenization theory for our model problem (2.1). These results are standard and can be found in [6, 18, 8, 3, 27]. We borrow the following definition from Definition 1.2.15 of [3].

Definition 2.1 (H-convergence). A sequence of matrices $A^{\epsilon} \in L^{\infty}\left(\Omega ; \mathcal{M}_{\alpha, \beta}\right)$ is said to $H$ converge to an H-limit, matrix $A^{*}(x) \in L^{\infty}\left(\Omega ; \mathcal{M}_{\alpha, \beta}\right)$ if, for any right-hand side $f \in H^{-1}(\Omega)$, the sequence $u^{\epsilon}$ of the solutions of

$$
\begin{aligned}
& -\nabla \cdot\left(A^{\epsilon}(x) \nabla u^{\epsilon}\right)=f \quad \text { in } \quad \Omega, \\
& u^{\epsilon}=0 \text { on } \partial \Omega \text {. }
\end{aligned}
$$

satisfies

$$
\begin{aligned}
& u^{\epsilon} \rightarrow u^{*} \text { weakly in } H_{0}^{1}(\Omega) ; \\
& A^{\epsilon} \nabla u^{\epsilon} \rightarrow A^{*} \nabla u^{*} \text { weakly in } L^{2}(\Omega)^{d},
\end{aligned}
$$

where $u^{*}$ is the solution of the homogenized equation

$$
\begin{array}{rllll}
-\nabla \cdot\left(A^{*}(x) \nabla u^{*}\right) & = & f & \text { in } & \Omega, \\
u^{*} & = & 0 & \text { on } & \partial \Omega .
\end{array}
$$

Under such a condition, we have the following result (Theorem 1.2.16 of [3]).

TheOREM 2.2. Any sequence $A^{\epsilon} \in L^{\infty}\left(\Omega ; \mathcal{M}_{\alpha, \beta}\right)$ has a subsequence, still denoted by $A^{\epsilon}$, and a homogenized matrix $A^{*} \in L^{\infty}\left(\Omega ; \mathcal{M}_{\alpha, \beta}\right)$ such that $A^{\epsilon} H$-converges to $A^{*}$.

Define $d$ sequences of oscillating test functions $w_{i}^{\epsilon}, 1 \leq i \leq d$, satisfying

$$
\begin{aligned}
w_{i}^{\epsilon} & \rightarrow x_{i} & & \text { weakly in } H^{1}(\Omega) ; \\
g_{u}^{\epsilon}=-\nabla \cdot\left(A^{\epsilon} w_{i}^{\epsilon}\right) & \rightarrow g_{i}=-\nabla \cdot\left(A^{*} e_{i}\right) & & \text { strongly in } H^{-1}(\Omega) .
\end{aligned}
$$

where $e_{i}$ is the $i$ 'th canonical basis of $\mathbb{R}^{\mathrm{n}}$. Let $W^{\epsilon}=\nabla w^{\epsilon}$ with $w=\left(w_{1}^{\epsilon}, w_{2}^{\epsilon}, \cdots, w_{d}^{\epsilon}\right)$ be the so called corrector matrix (Section 1.3.6 of [3]). Then we have

$$
\nabla u^{\epsilon}=W^{\epsilon} \nabla u^{*}+r^{\epsilon}, \quad \text { with } r^{\epsilon} \rightarrow 0 \text { strongly in } L_{l o c}^{1}(\Omega)^{d} .
$$

For a smoother $u^{*} \in H^{2}(\Omega)$, we have (Remark 1.3.40 of [3]),

$$
u^{\epsilon}=u^{*}+\sum_{i=1}^{d}\left(w_{i}^{\epsilon}-x_{i}\right) \frac{\partial u^{*}}{\partial x_{i}}+r^{\epsilon}
$$

with

$$
r^{\epsilon} \rightarrow 0 \quad \text { strongly in } W_{l o c}^{1,1}(\Omega) .
$$

The above homogenization theory with oscillating test functions is the basis for many numerical methods, e.g., in [22], a globally solved oscillating test function is used to define the numerical method. In this paper, we will use local versions of oscillating test functions to build multiscale finite element elements, following the ideas of [4] and [14.

In the periodic case we have

$$
A^{\epsilon}(x)=A\left(\frac{x}{\epsilon}\right),
$$


where $y \mapsto A(y)$ is a $Y$-periodic function with $Y=(0,1)^{d}$.

Let $\chi_{i}, i=1, \cdots, d$, be the solution to the cell problem

$$
\begin{cases}-\operatorname{div}_{y}\left(A(y) \nabla_{y} \chi_{i}\right)=\operatorname{div}_{y}\left(A(y) e_{i}\right) & \text { in } Y, \\ y \mapsto x_{i}(y) & Y \text { - periodic. }\end{cases}
$$

The notation $\langle\cdot\rangle_{Y}$ is used to denote the mean of a function in domain $Y$ :

$$
\langle f\rangle_{Y}=\frac{1}{|Y|} \int_{Y} f(x) d x .
$$

Notice that $\chi_{i}$ here is $w_{i}^{\epsilon}-x_{i}$ and defined for general cases. We then have the following approximation results

$$
u^{\epsilon}(x) \approx u^{*}(x)+\epsilon \sum_{i=1}^{n} \chi_{i}\left(\frac{x}{\epsilon}\right) \frac{\partial u^{*}}{\partial x_{i}}(x)
$$

and

$$
\nabla u^{\epsilon}(x) \approx \nabla u^{*}(x)+\epsilon \sum_{i=1}^{n}\left(\nabla_{y} \chi_{i}\right)\left(\frac{x}{\epsilon}\right) \frac{\partial u^{*}}{\partial x_{i}}(x) .
$$

Here $u^{*}$ is the solution of the homogenization problem:

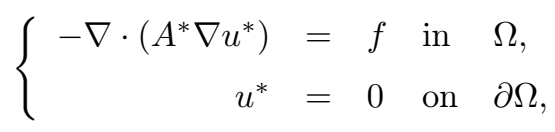

where $A^{*}$ is a constant homogenized matrix given by the explicit formula

$$
A^{*} e_{i}=\int_{Y} A(y)\left(e_{i}+\nabla_{y} \chi_{i}\right) d y
$$

3. Multiscale finite element methods based on local oscillating test functions. In this section, we define MsFEM in the spirit of [4, 14]. We first solve to obtain oscillating test functions on the local elements or on larger patches if oversampling technique is used. The multiscale finite element then is defined by a composite rule [4] or by a more direct approach [14]. Oversampling and Petrov-Galerkin formulations can be used to improve the accuracy of the methods. Compared with the approach originally introduced in [16, this approach is natural for the extension to high-order multiscale finite element methods [4, 14] and easier to adapt to take advantage of reduced basis acceleration of multiscale finite element method on general meshes.

Let $\mathcal{T}_{h}$ be a regular finite element mesh of $\Omega$. We first introduce the local problem to solve for the local oscillating test functions: For each $K \in \mathcal{T}_{h}$, define $\hat{w}_{i}^{\epsilon, K}, i=1, \cdots, d$, as the solution of

$$
\left\{\begin{aligned}
-\nabla \cdot\left(A^{\epsilon} \nabla \hat{w}_{i}^{\epsilon, K}\right) & =0 \quad \text { in } \quad K, \\
\hat{w}_{i}^{\epsilon, K} & =x_{i} \text { on } \partial K .
\end{aligned}\right.
$$

For each $K \in \mathcal{T}_{h}$, a quasi-uniform fine mesh $\mathcal{T}_{h^{\prime}}(K)$ with element size $h^{\prime}$ is used and we always assume that the following inequality holds

$$
0<h^{\prime}<\epsilon<h<1
$$

relating the coarse mesh size $h$, the period $\epsilon$, and the local mesh size $h^{\prime}$, where $\epsilon$ is the characteristic size of the fine scale. 
Define

$$
W_{h^{\prime}}(K)=\left\{w \in C^{0}(K):\left.w\right|_{T} \in P_{k^{\prime}}(T), \forall T \in \mathcal{T}_{h^{\prime}}(K)\right\},
$$

and let $w_{i}^{\epsilon, K}$ be the $P_{k^{\prime}}$ finite element approximation of $\hat{w}_{i}^{\epsilon, K}$ in 3.1 using mesh $\mathcal{T}_{h^{\prime}}^{K}$. Then the local finite element approximation for the oscillating test function is: Find $w_{i}^{\epsilon, K} \in W_{h^{\prime}}(K)$, $w_{i}^{\epsilon, K}=x_{i}$ on $\partial K$, such that

$$
\left(A^{\epsilon} \nabla \hat{w}_{i}^{\epsilon, K}, \nabla v\right)=0, \quad v \in W_{h^{\prime}}(K) \cap H_{0}^{1}(K) .
$$

Define $w^{\epsilon, h}=\left(w_{1}^{\epsilon, h}, \cdots, w_{d}^{\epsilon, h}\right) \in H^{1}(\Omega)^{d}$, where $w_{i}^{\epsilon, h} \in H^{1}(\Omega)$ with $w_{i}^{\epsilon, h}=w_{i}^{\epsilon, K}$ for each $K \in \mathcal{T}_{h}$.

To remove the resonance effect [9] associated with the lack of correct boundary conditions for the local problem, we use an oversampling method on a larger domain $S \supset K$. Define $\hat{w}_{i}^{\epsilon, S}$, $i=1, \cdots d$, as the solution of

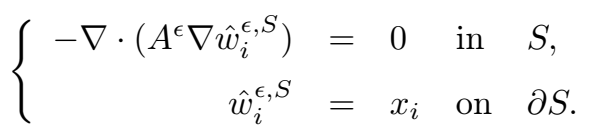

We define $\hat{w}_{i}^{\epsilon, K}=\left.\hat{w}_{i}^{\epsilon, S}\right|_{K}, i=1, \cdots, d$, and $\hat{w}^{\epsilon}$ accordingly. In general, $\hat{w}^{\epsilon}$, obtained from the restriction of the oversampling method, is not in $H^{1}(\Omega)^{n}$.

For a polygonal domain $S \supset K$, define a quasi-uniform fine mesh $\mathcal{T}_{h^{\prime}}(S)$ with element size $h^{\prime}$. The edges of the mesh are aligned with the sides of $K$. Define

$$
W_{h^{\prime}}(S)=\left\{w \in C^{0}(S):\left.w\right|_{T} \in P_{k^{\prime}}(T), \forall T \in \mathcal{T}_{h^{\prime}}(S)\right\},
$$

and let $w_{i}^{\epsilon, S}$ be the $P_{k^{\prime}}$ finite element approximation of $\hat{w}_{i}^{\epsilon, S}$ of 3.4 using mesh $\mathcal{T}_{h^{\prime}}(S)$. Find $w_{i}^{\epsilon, S} \in W_{h^{\prime}}(S), w_{i}^{\epsilon, S}=x_{i}$ on $\partial S$, such that

$$
\left(A^{\epsilon} \nabla \hat{w}_{i}^{\epsilon, S}, \nabla v\right)=0, \quad \forall v \in W_{h^{\prime}}(S) \cap H_{0}^{1}(S) .
$$

With $w^{\epsilon, K}=\left.w^{\epsilon, S}\right|_{K}$, we can define $w^{\epsilon, h}$ accordingly. Like $\hat{w}^{\epsilon}, w^{\epsilon, h}$ is generally not an $H^{1}$ vector function.

3.1. MsFEM introduced in [4]. Denote the $P_{k}$ conforming finite element space associated with the triangulation $\mathcal{T}$ by

$$
V_{h}=\left\{v \in H_{0}^{1}(\Omega):\left.v\right|_{K} \in P_{k}: \forall K \in \mathcal{T}_{h}\right\} .
$$

When a quadrilateral mesh is used,

$$
V_{h}=\left\{v \in H_{0}^{1}(\Omega):\left.v\right|_{K} \in Q_{k}: \forall K \in \mathcal{T}_{h}\right\} .
$$

Let $\left(\Phi_{\ell}^{h}\right)_{\ell=1, \cdots, N_{h}}$ denote a finite element basis of $V_{h}$, where $N_{h}=\operatorname{dim} V_{h}$. Define the conforming multiscale finite element basis introduced in [4],

$$
\Phi_{c, \ell}^{\epsilon, h}(x)=\Phi_{\ell}^{h}\left(w^{\epsilon, h}(x)\right), \quad \ell=1, \cdots, N_{h},
$$

and associated conforming multiscale finite element space [4] as

$$
V_{c, h}^{\epsilon}=\operatorname{span}\left\{\Phi_{c, \ell}^{\epsilon, h}\right\}_{\ell=1, \cdots, N_{h}} .
$$

The Galerkin multiscale finite element method is to seek $u_{h}^{\epsilon} \in V_{c, h}^{\epsilon}$, such that

$$
a\left(u_{h}^{\epsilon}, v_{h}^{\epsilon}\right)=f\left(v_{h}^{\epsilon}\right), \quad \forall v_{h}^{\epsilon} \in V_{c, h}^{\epsilon} .
$$




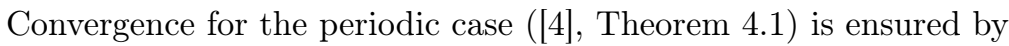

TheOrem 3.1. Assume that the coefficient tensor is periodic, i.e., $A^{\epsilon}=a\left(\frac{x}{\epsilon}\right)$. Let $u^{\epsilon}$ be the exact solution of the original problem (2.2) and $u_{h}^{\epsilon}$ be the solution of (3.6). Assume that $u^{*} \in W^{k+1, \infty}(\Omega)$ and $\chi_{i} \in W^{1, \infty}(Y)$. Then there exists a constant $C$ independent of $\epsilon$ and $h$ such that

$$
\left\|\nabla\left(u^{\epsilon}-u_{h}^{\epsilon}\right)\right\|_{0} \leq C\left(h^{k}\|f\|_{0}+\sqrt{\frac{\epsilon}{h}}+\left(\frac{h^{\prime}}{\epsilon}\right)^{k^{\prime}}\right) .
$$

The Petrov-Galerkin multiscale finite element method for the problem is obtained by seeking $u_{h}^{\epsilon} \in V_{c, h}^{\epsilon}$, such that

$$
a\left(u_{h}^{\epsilon}, v_{h}\right)=f\left(v_{h}\right), \quad \forall v_{h} \in V_{h} .
$$

Convergence for the periodic case stated by

Theorem 3.2. Assume that the coefficient tensor is periodic, i.e., $A^{\epsilon}=a\left(\frac{x}{\epsilon}\right)$, and assume that the size of over-sampling domain is large enough such that Assumption 2.1 of [17] is true. Then (3.8) is well-posed. Let $u^{\epsilon}$ be the exact solution of the original problem (2.2) and $u_{h}^{\epsilon}$ be the solution of (3.8). Assume that $u^{*} \in W^{k+1, \infty}(\Omega)$ and $\chi_{i} \in W^{1, \infty}(Y)$. There exists a constant $C$ independent of $\epsilon$ and $h$ such that

$$
\left\|\nabla\left(u^{\epsilon}-u_{h}^{\epsilon}\right)\right\|_{0} \leq C\left(h^{k}\|f\|_{0}+\sqrt{\epsilon}+\left(\frac{h^{\prime}}{\epsilon}\right)^{k^{\prime}}\right) .
$$

This theorem can be proved in a fashion similar to the convergence theorem in [14.

3.2. MsFEM introduced in [14]. For a function $v \in H^{1}(\Omega)$, introduce a map $J^{\epsilon, h}$ such that

$$
\left.J^{\epsilon, h} v\right|_{K}=\left.\left(v+\left(w^{\epsilon, h}-x\right) \cdot \nabla v\right)\right|_{K} \text { on each } K \in \mathcal{T}_{h} .
$$

If we define

$$
\Phi_{n c, \ell}^{\epsilon, h}=J^{\epsilon, h} \Phi_{\ell}^{h}, \quad \ell=1, \cdots, N_{h},
$$

then the multiscale finite element space, introduced in [14] is defined as

$$
V_{n c, h}^{\epsilon}=\operatorname{span}\left\{\Phi_{n c, \ell}^{\epsilon, h}\right\}_{\ell=1, \cdots, N_{h}} .
$$

Note that $V_{n c, h}^{\epsilon} \not \subset H_{0}^{1}(\Omega)$ if oversampling is used. We introduce the following bilinear form:

$$
a_{h}(u, v)=\sum_{K \in \mathcal{T}}\left(A^{\epsilon} \nabla u, \nabla v\right)_{K}
$$

Note that if $u$ and $v$ are both in $H_{0}^{1}(\Omega)$, then $a_{h}(u, v)=a(u, v)$. The Galerkin multiscale finite element method seeks $u_{h}^{\epsilon} \in V_{n c, h}^{\epsilon}$, such that

$$
a_{h}\left(u_{h}^{\epsilon}, v_{h}^{\epsilon}\right)=f\left(v_{h}^{\epsilon}\right), \quad \forall v_{h}^{\epsilon} \in V_{n c, h}^{\epsilon} .
$$

As some functions to be considered are not in $H_{0}^{1}(\Omega)$, but only in $H^{1}(K)$ for $K \in \mathcal{T}_{h}$, let us define an equivalent broken $H^{1}$-norm as

$$
\|v\|_{h, \Omega}=\left(\sum_{K \in \mathcal{T}_{h}}\|\nabla v\|_{0, K}^{2}\right)^{1 / 2} .
$$


In the spirit of [4, 10, 14, we can prove the following convergence theorem

Theorem 3.3. Assume that the coefficient tensor is periodic, i.e., $A^{\epsilon}=a\left(\frac{x}{\epsilon}\right)$. Let $u^{\epsilon}$ be the exact solution of the original problem (2.2) and $u_{h}^{\epsilon}$ be the solution of (3.12). Assume that $u^{*} \in W^{k+1, \infty}(\Omega)$ and $\chi_{i} \in W^{1, \infty}(Y)$. Then there exists a constant $C$ independent of $\epsilon$ and $h$ such that

$$
\left\|u^{\epsilon}-u_{h}^{\epsilon}\right\|_{h} \leq C\left(h^{k}\|f\|_{0}+\sqrt{\frac{\epsilon}{h}}+\left(\frac{h^{\prime}}{\epsilon}\right)^{k^{\prime}}\right) .
$$

As in the previous case, we can also define a Petrov-Galkerin multiscale finite element method: Find $u_{h}^{\epsilon} \in V_{n c, h}^{\epsilon}$, such that

$$
a_{h}\left(u_{h}^{\epsilon}, v_{h}\right)=f\left(v_{h}\right), \quad \forall v_{h} \in V_{h} .
$$

The following convergence result can be found in [14].

TheOrem 3.4. Assume that the coefficient tensor is periodic, i.e., $A^{\epsilon}=a\left(\frac{x}{\epsilon}\right)$ and assume that the size of the over-sampling domain is large enough such that Assumption 2.1 of [17] holds. Then the discrete problem (3.15) is well-posed. Let $u^{\epsilon}$ be the exact solution of the original problem (2.2) and $u_{h}^{\epsilon}$ be the solution of (3.8). Assume that $u^{*} \in W^{k+1, \infty}(\Omega)$ and $\chi_{i} \in W^{1, \infty}(Y)$. Then there exists a constant $C$ independent of $\epsilon$ and $h$ such that

$$
\left\|u^{\epsilon}-u_{h}^{\epsilon}\right\|_{h} \leq C\left(h^{k}\|f\|_{0}+\sqrt{\epsilon}+\left(\frac{h^{\prime}}{\epsilon}\right)^{k^{\prime}}\right) .
$$

4. RB-MsFEM for uniform rectangular meshes. In this section, we discuss the use of the reduced basis method for MsFEM on uniform rectangular meshes. With a uniform rectangular finite element mesh, each element is a rectangle of the same size and the coefficient matrix in each element is similar. In this case, it is expected that the oscillating test functions across these elements have similar patterns and can be approximated by a compact reduced basis. For a rectangular element $K$ with the center $x_{K}$ and the size $h_{1} \times \cdots \times h_{d}$, we denote $h=\operatorname{diag}\left(h_{1}, \cdots, h_{d}\right)$.

4.1. Parameterization of the Oscillating functions. The local problem for the oscillating test functions is: Find $w=\left(w_{1}, \cdots, w_{d}\right) \in H^{1}(K)^{d}$, such that

$$
-\nabla \cdot\left(A^{\epsilon}(x) \nabla w_{i}\right)=0 \quad \text { in } \quad K \quad \text { and } \quad w_{i}=x_{i}, \quad i=1, \cdots, d .
$$

Let $q_{i}=w_{i}-x_{i}, i=1, \cdots, d$. Then $q_{i}$ satisfies

$$
-\nabla \cdot\left(A^{\epsilon}(x) \nabla q_{i}\right)=\nabla \cdot\left(A^{\epsilon}(x) e_{i}\right) \quad \text { in } \quad K \quad i=1, \cdots, d,
$$

where $e_{i}, i=1, \cdots, d$ is the canonical basis of $\mathbb{R}^{\mathrm{d}}$.

The variational problem is: Seek $q_{i} \in H_{0}^{1}(K), i=1, \cdots, d$, such that

$$
\left(A^{\epsilon}(x) \nabla q_{i}, \nabla v\right)_{K}=-\left(A^{\epsilon}(x) e_{i}, \nabla v\right)_{K} \quad \forall v \in H_{0}^{1}(K) .
$$

A key step of the reduced basis method is to parameterize the problem to be solved. We introduce the parameterization of the element $K$ centered at $x_{K}$ by $x=x_{K}+h z$, with $z \in Z=$ $(-1 / 2,1 / 2)^{d}$. Let $A\left(x_{K}, z\right)=A^{\epsilon}(x)=A^{\epsilon}\left(x_{K}+h z\right)$, and let $\hat{q}_{i}\left(x_{K}, z\right)=q_{i}(x)=q_{i}\left(x_{K}+h z\right)$, then $\hat{q}_{i}\left(x_{K}, z\right) \in H_{0}^{1}(Z)$ satisfies

$$
\left(A\left(x_{K}, z\right) \nabla \hat{q}_{i}\left(x_{K}, z\right), \nabla v(z)\right)_{Y}=-\left(A\left(x_{K}, z\right) e_{i}, \nabla v(z)\right)_{Y} \quad \forall v \in H_{0}^{1}(Z) .
$$


We can recover the original function $q_{i}$ as $q_{i}(x)=\hat{q}_{i}\left(x_{K}, h^{-1}\left(x-x_{K}\right)\right)$.

For coefficient matrices without explicit periodicity, we have to use Dirichlet boundary conditions for the local problems and use the method described above. A simple example of such a coefficient matrix is

$$
A^{\epsilon}(x)=\frac{1}{4+\sin \left(x_{1} / \epsilon_{1}\right)+\sin \left(\sqrt{2} x_{1} / \epsilon_{1}\right)} \cdot I
$$

However, for coefficient matrices with explicit periodicity, we should take advantage of it and use more accurate periodic boundary conditions. Note that periodic boundary conditions can be viewed as having the equation defined in the whole space. In this case, the solution is accurate and does not suffer from the resonance error.

Let's consider a few special cases of $A^{\epsilon}(x)$. Assume $A^{\epsilon}(x)=a(x / \epsilon)$, with $\epsilon=\left(\epsilon_{1}, \cdots, \epsilon_{d}\right)$, with the entries of the matrix $a$ being periodic functions with period $\epsilon_{i}$ in the direction of $x_{i}$, $i=1, \cdots, d$, e.g.,

$$
a(x / \epsilon)=\frac{1}{\left(2+\sin \left(x_{1} / \epsilon_{1}\right)\right)\left(2+\sin \left(x_{2} / \epsilon_{2}\right)\right)} I
$$

for $d=2$. In this case, we can choose the size of element $H_{i}=\epsilon_{i}, i=1, \cdots, d$. Obviously, we can also choose $H_{i}=n \epsilon_{i}$ with $n$ a positive integer, but $n=1$ is the simplest and most economical choice. Since the mesh is aligned with the period the matrix $A^{\epsilon}$ is identical within each element. Thus, only one local problem needs to be solved for all elements and, instead of the Dirichlet boundary condition as in the general case, the periodic boundary condition should be used to improve accuracy. For such a case, a reduced basis method is not needed.

Define $\hat{H}_{p e r}^{1}(K)=\left\{v \in H^{1}(K) \mid v\right.$ periodic in $\left.K, \int_{K} v d x=0\right\}$. The local problem is to find $q_{i} \in \hat{H}_{p e r}^{1}(K)$ that satisfies

$$
-\nabla \cdot\left(a(x / \epsilon) \nabla q_{i}\right)=\nabla \cdot\left(a(x / \epsilon) e_{i}\right) \quad \text { in } \quad K, \quad i=1, \cdots, d .
$$

The variational problem is seeking $q_{i} \in \hat{H}_{p e r}^{1}(K), i=1, \cdots, d$, such that

$$
\left(a(x / \epsilon) \nabla q_{i}, \nabla v\right)_{K}=-\left(a(x / \epsilon) e_{i}, \nabla v\right)_{K}, \quad \forall v \in \hat{H}_{p e r}^{1}(K) .
$$

We can recover the local oscillating test function $w$ by letting $w=x+q$.

The second special case is for $A^{\epsilon}(x)=a(x, x / \epsilon)$, where the entries of the matrix $a$ are periodic functions with period $p_{i}$ in the direction of $x_{i}$ of the second variable, $i=1, \cdots, d$. An example could be

$$
a(x, x / \epsilon)=\frac{1}{\left(4+x_{1}+\sin \left(x_{1} / \epsilon_{1}\right)\right)\left(4+x_{2}+\sin \left(x_{2} / \epsilon_{2}\right)\right)} I
$$

for $x \in[0,1]^{2}$. We choose the size of element $H_{i}=\epsilon_{i}, i=1, \cdots, d$. Since we ensure that the mesh is aligned with the period in each element, the location of the center $x_{K}$ of the element $K$ is identical in the period of the second variable. As in the first case, the periodic boundary condition should be used to obtain a solution of optimal accuracy.

The local problem is to find $q_{i} \in \hat{H}_{\text {per }}^{1}(K)$ that satisfies

$$
-\nabla \cdot\left(a(x, x / \epsilon) \nabla q_{i}\right)=\nabla \cdot\left(a(x, x / \epsilon) e_{i}\right) \quad \text { in } \quad K, \quad i=1, \cdots, d .
$$

The variational problem is to seek $q_{i} \in \hat{H}_{p e r}^{1}(K), i=1, \cdots, d$, such that

$$
\left(a(x, x / \epsilon) \nabla q_{i}, \nabla v\right)_{K}=-\left(a(x, x / \epsilon) e_{i}, \nabla v\right)_{K}, \quad \forall v \in \hat{H}_{p e r}^{1}(K) .
$$


With the parameterization $x=x_{K}+h z, z \in Z=(-1 / 2,1 / 2)^{d}$, let $A\left(x_{K}, z\right)=a\left(x_{K}+h z, z\right)$, and let $\hat{q}_{i}\left(x_{K}, z\right)=q_{i}(x)=q_{i}\left(x_{K}+h z\right)$. Then $\hat{q}_{i}\left(x_{K}, \cdot\right) \in \hat{H}_{p e r}^{1}(Z)$ satisfies

$$
\left(A\left(x_{K}, z\right) \nabla \hat{q}_{i}\left(x_{K}, z\right), \nabla v(z)\right)_{Y}=-\left(A\left(x_{K}, z\right) e_{i}, \nabla v(z)\right)_{Y} \quad \forall v \in \hat{H}_{p e r}^{1}(Z) .
$$

We can get $w$ in a fashion similar to the general case.

Since the first variable of $a(x, x / \epsilon)$ is a slow variable, we can use $x_{K}$ at the element center to approximate the function without losing accuracy, provided only that the element size of $K$ is reasonably small. The local problem now is: Seek $\hat{q}_{i}\left(x_{K}, \cdot\right) \in \hat{H}_{\text {per }}^{1}(Z)$ such that

$$
\left(a\left(x_{K}, z\right) \nabla \hat{q}_{i}\left(x_{K}, z\right), \nabla v(z)\right)_{Y}=-\left(a\left(x_{K}, z\right) e_{i}, \nabla v(z)\right)_{Y} \quad \forall v \in \hat{H}_{p e r}^{1}(Z) .
$$

This simplification is useful for the reduced basis method as a more complicated non-affine coefficient will require more terms in its empirical interpolation and, hence, impact the computational efficiency of the method.

4.2. Finite element approximation of the local parameterized problem. In this subsection, we introduce the finite element approximation of the local parametrized problem (4.4), i.e., for $i=1, \cdots, d$, seek $\hat{q}_{i}\left(x_{K}, z\right) \in H_{0}^{1}(Z)$, such that

$$
\left(A\left(x_{K}, z\right) \nabla \hat{q}_{i}\left(x_{K}, z\right), \nabla v(z)\right)_{Z}=-\left(A\left(x_{K}, z\right) e_{i}, \nabla v(z)\right)_{Z} \quad \forall v \in H_{0}^{1}(Z) .
$$

or

$$
a_{Z}\left(\hat{q}_{i}\left(x_{K}\right), v ; x_{K}\right)=\ell_{Y}\left(v ; x_{K}\right) \quad \forall v \in H_{0}^{1}(Y) .
$$

where

$$
a_{Z}\left(u\left(x_{K}\right), v ; x_{K}\right)=\left(A\left(x_{K}\right) \nabla u\left(x_{K}\right), \nabla v\right)_{Z}, \quad \ell_{Y}\left(v ; x_{K}\right)=-\left(A\left(x_{K}\right) e_{i}, \nabla v\right)_{Z},
$$

with $A\left(x_{K}\right)=A\left(x_{K}, z\right)$.

Define a triangulation $\mathcal{T}(Z)$ of $Z$. We assume the mesh is fine enough, i.e, when the mesh is mapped back to the original domain, the mapped mesh size of finite element space on $Z$ should be smaller than the characteristic size $\epsilon$. Define

$$
X^{f e}(Z)=\left\{v \in H_{0}^{1}(Z):\left.v\right|_{T} \in P_{k^{\prime}}(T), \quad \forall T \in \mathcal{T}(Z)\right\}
$$

For $i=1, \cdots, d$, let $\hat{q}_{i}^{f e}\left(x_{K}, z\right) \in X^{f e}(Z)$ be the finite element approximation of $\hat{q}_{i}\left(x_{K}, z\right)$, such that

$$
a_{Z}\left(\hat{q}_{i}^{f e}\left(x_{K}\right), v ; x_{K}\right)=\ell_{Y}\left(v ; x_{K}\right) \quad \forall v \in X^{f e}(Z) .
$$

The local finite element problem 4.12 is well-posed with a coercivity constant $\alpha\left(x_{K}\right)=\min \lambda_{x_{K}}$, where $\lambda_{x_{K}}$ are the eigenvalues of $A\left(x_{K}, z\right)$. We have

$$
a_{Z}\left(v, v ; x_{K}\right) \geq \alpha_{x_{K}}\|\nabla v\|_{0, Z}^{2}, \quad v \in X^{f e}(Z) .
$$

The fast evaluation of reduced basis method depends critically on the affine representation of the coefficient matrix $A\left(x_{K}, z\right)$ at an element $K$. That is,

$$
A\left(x_{K}, z\right)=\sum_{q=1}^{Q} \Theta_{q}\left(x_{K}\right) A_{q}(z), \quad z \in Z .
$$

If the above affine representation is not available, we can use the empirical interpolation method (EIM) [5, 20] to recover an affine approximation of $A\left(x_{K}, z\right)$. 
We choose the parameter domain $\mathcal{D}$ to be the collection of $x_{K}$ for all $K$ in the uniform rectangular mesh $\mathcal{T}$. This discrete parameter domain is the most economical choice of the parameter domain. For each $i=1, \cdots, d$, we seek an collection of $N_{i}$ parameters $S_{N_{i}}=\left\{x_{K, i}^{1}, \cdots, x_{K, i}^{N_{i}}\right\}$ in the parameter domain $\mathcal{D}$. The corresponding reduced basis space is $X_{i, N}^{r b}=\operatorname{span}\left\{\hat{q}_{i}^{f e}\left(x_{K, i}^{1}\right), \cdots, \hat{q}_{i}^{f e}\left(x_{K, i}^{N_{i}}\right)\right\}$, where $\hat{q}_{i}^{f e}\left(x_{K, i}^{j}\right)$ is the numerical solution of problem 4.12 for the parameter values $x_{K, i}^{j}$, for $1 \leq j \leq N_{i}, 1 \leq i \leq d$.

The reduced basis approximation of problem (4.4) with index $i, i=1, \cdots, d$ is then defined as: For an $x_{K} \in \mathcal{D}$, find $\hat{q}_{i}^{r b}\left(x_{K}\right) \in X_{i, N}^{r b}$ such that

$$
a_{Z}\left(\hat{q}_{i}^{r b}\left(x_{K}\right), v ; x_{K}\right)=\ell_{Z}\left(v ; x_{K}\right), \quad \forall v \in X_{i, N}^{r b},
$$

Due to the affine assumption (4.13), an efficient offline-online strategy is immediately possible, see 23 .

In order to build the parameter set $S_{N_{i}}$ and the corresponding reduced basis space $X_{i, N}^{r b}$, a greedy algorithm based on a posteriori residual-driven error estimator is used during the offline stage. Let us define the error function

$$
e_{i}\left(x_{K}\right)=\hat{q}_{i, N}^{r b}\left(x_{K}\right)-\hat{q}_{i}^{f e}\left(x_{K}\right) \in X^{f e}(Z)
$$

as the difference between the reduced basis solution $\hat{q}_{i, N}^{r b}\left(x_{K}\right)$ and the finite element solution $\hat{q}_{i}^{f e}\left(x_{K}\right)$.

The residual $r\left(v ; x_{K}\right) \in\left(X^{f e}(Z)\right)^{\prime}$ is defined as

$$
r\left(v ; x_{K}\right):=\ell_{Z}\left(v ; x_{K}\right)-a_{Z}\left(\hat{q}_{i}^{r b}\left(x_{K}\right), v ; x_{K}\right), \quad \forall v \in X^{f e}(Z),
$$

and its norm as

$$
\left\|r\left(\cdot ; x_{K}\right)\right\|_{\left(X^{f e}(Z)\right)^{\prime}}:=\sup _{v \in X^{f e}(Z)} \frac{r\left(v ; x_{K}\right)}{\|\nabla v\|_{0, Z}} .
$$

We then define the estimator as

$$
\eta\left(N ; x_{K}\right):=\frac{\left\|r\left(\cdot ; \mathbf{X}_{K}\right)\right\|_{\left(X^{f e}(Z)\right)^{\prime}}}{\alpha\left(x_{K}\right)} .
$$

The training set $\Xi_{\text {train }}=\mathcal{D}$ is defined since $\mathcal{D}$ is discrete. Let us suppose we already have $S_{i, N}$ and the corresponding $X_{i, N}^{r b}$. We choose

$$
x_{K, i}^{N+1}:=\operatorname{argmax}_{x_{K} \in \Xi_{\text {train }}} \eta\left(N ; x_{K}\right),
$$

as the next sample point and let $S_{N+1}:=S_{N} \cup\left\{x_{K, i}^{N+1}\right\}$. This allows building the corresponding space $X_{i, N+1}^{r b}$ in a progressive fashion, repeated repeated until $N$ is large enough to ensure that $\max _{\boldsymbol{\mu} \in \Xi_{\text {train }}} \eta\left(N ; x_{K}\right)$ is less than a predefined tolerance $\delta$.

When the basis is built, we can use 4.14 to compute the reduced basis solution $\hat{q}_{i}^{r b}$ with a computational cost of $O\left(N_{i}^{3}\right)$. This allows for the recovery of the local oscillating test function $w_{i}^{r b}$ by letting $w_{i}^{r b}=q_{i}^{r b}+x_{i}=\hat{q}_{i}^{r b}\left(x_{K}, z\right)+x_{i}$ and define the RB-based conforming multiscale finite element basis in the spirit of [4] as

$$
\Phi_{c, \ell}^{\epsilon, h, r b}=\Phi_{\ell}^{h}\left(w^{\epsilon, r b}(x)\right) .
$$

The conforming reduced basis multiscale finite element space is defined as

$$
V_{c, h}^{\epsilon, r b}=\operatorname{span}\left\{\Phi_{c, \ell}^{\epsilon, r b}\right\}_{\ell=1, \cdots, N_{h}} .
$$


The Galerkin RB-MsFEM of the problem is: Seek $u_{h}^{\epsilon, r b} \in V_{c, h}^{\epsilon, r b}$, such that

$$
a\left(u_{h}^{\epsilon, r b}, v_{h}^{\epsilon}\right)=f\left(v_{h}^{\epsilon}\right), \quad \forall v_{h}^{\epsilon} \in V_{c, h}^{\epsilon, r b} .
$$

With the affine assumption on $A^{\epsilon}$ and $f$, we can pre-compute the matrix and vector entries in the offline stage. During the online stage, the computational cost reduces to building both sides of 4.20, which is independent of the local DOFs on $K$. Similar procedures can be found in [23, 21.

Since the domain of parameter $\mathcal{D}$ is of finite cardinality, the following error estimation follows

$$
\left\|\nabla\left(w^{\epsilon, h}-w^{\epsilon, r b}\right)\right\|_{0, K} \leq \delta, \quad \forall K \in \mathcal{T}_{h}
$$

Summing up for all elements $K \in \mathcal{T}_{h}$, we have

$$
\left\|\nabla\left(w^{\epsilon, h}-w^{\epsilon, r b}\right)\right\|_{0, \Omega} \leq \sqrt{N_{h}} \delta .
$$

For a sufficiently smooth function $v$ with meaningful nodal values, define $\Pi_{h}$ to be the $V_{h^{-}}$ interpolation operator,

$$
\Pi_{h} v=\sum_{\ell=1}^{N_{h}} v\left(n_{\ell}\right) \Phi_{\ell}^{h} \in V_{h}
$$

Also define $\Pi_{c, h}^{\epsilon}$ to be the $V_{c, h}^{\epsilon}$-interpolation operator,

$$
\Pi_{c, h}^{\epsilon} v=\sum_{\ell=1}^{N_{h}} v\left(n_{\ell}\right) \Phi_{c, \ell}^{\epsilon, h}=\sum_{\ell=1}^{N_{h}} v\left(n_{\ell}\right) \Phi_{\ell}^{h}\left(w^{\epsilon, h}\right) \in V_{c, h}^{\epsilon},
$$

and $\Pi_{c, h}^{\epsilon, r b}$ as the $V_{c, h}^{\epsilon, r b}$-interpolation operator,

$$
\Pi_{c, h}^{\epsilon, r b} v=\sum_{\ell=1}^{N_{h}} v\left(n_{\ell}\right) \Phi_{c, \ell}^{\epsilon, h, r b}=\sum_{\ell=1}^{N_{h}} v\left(n_{\ell}\right) \Phi_{\ell}^{h}\left(w^{\epsilon, r b}\right) \in V_{c, h}^{\epsilon, r b} .
$$

THEOREM 4.1. Let $u^{\epsilon}$ be the solution of 2.2) and $u_{h}^{\epsilon, r b}$ be the RB-MsFEM solution of (4.20). There exists a positive constant $C$ independent of $\epsilon$ and $h$, such that

$$
\left\|\nabla\left(u^{\epsilon}-u_{h}^{\epsilon, r b}\right)\right\|_{0} \leq C\left(h^{k}+\sqrt{\frac{\epsilon}{h}}+\left(\frac{h^{\prime}}{\epsilon}\right)^{k^{\prime}}+\sqrt{N_{h}} \delta\right) .
$$

Proof. From Céa's lemma, there exists a constant $C$ independent of $\epsilon$ and $h$ such that

$$
\left\|\nabla\left(u^{\epsilon}-u_{h}^{\epsilon, r b}\right)\right\|_{0} \leq C \inf _{v \in V_{c, h}^{\epsilon, r b}}\left\|\nabla\left(u^{\epsilon}-v\right)\right\|_{0}
$$

Choose $v=\Pi_{c, h}^{\epsilon, r b} u^{*}$. By the triangle inequality, we have

$$
\left\|\nabla\left(u^{\epsilon}-v\right)\right\|_{0} \leq\left\|\nabla\left(u^{\epsilon}-\Pi_{c, h}^{\epsilon} u^{*}\right)\right\|_{0}+\left\|\nabla\left(\Pi_{c, h}^{\epsilon} u^{*}-\Pi_{c, h}^{\epsilon, r b} u^{*}\right)\right\|_{0}
$$

where $u_{h}^{\epsilon}$ is the solution of (3.6) and $u^{*}$ is the homogenized solution. We have the upper bound of the first term of the right-hand side by the proof of Theorem 4.1 of [4]:

$$
\left\|\nabla\left(u^{\epsilon}-\Pi_{c, h}^{\epsilon} u^{*}\right)\right\|_{0} \leq C\left(h^{k}+\sqrt{\frac{\epsilon}{h}}+\left(\frac{h^{\prime}}{\epsilon}\right)^{k^{\prime}}\right) .
$$


The second term on the right-hand side can be bounded

$$
\begin{aligned}
\left\|\nabla\left(\Pi_{c, h}^{\epsilon} u^{*}-\Pi_{c, h}^{\epsilon, r b} u^{*}\right)\right\|_{0} & =\left\|\nabla\left(\Pi_{h} u^{*}\left(w^{\epsilon, h}\right)-\Pi_{h} u^{*}\left(w^{\epsilon, r b}\right)\right)\right\|_{0} \\
& \left.=\| \nabla\left(w^{\epsilon, h}-w^{\epsilon, r b}\right) \nabla\left(\Pi_{h} u^{*}\right) \circ\left(w^{\epsilon, h}-w^{\epsilon, r b}\right)\right) \|_{0} \\
& \leq\left\|u^{*}\right\|_{W^{1, \infty}}\left\|\nabla\left(w^{\epsilon, h}-w^{\epsilon, r b}\right)\right\|_{0} \leq C \sqrt{N_{h}} \delta .
\end{aligned}
$$

Thus the proof of the theorem.

When $\delta$ is small enough, we recover the same convergence result as for the standard method.

For a function $v \in H^{1}(\Omega)$, introduce a map $J^{\epsilon, r b}$, such that

$$
\left.J^{\epsilon, r b} v\right|_{K}=\left.\left(v+\left(w^{\epsilon, r b}-x\right) \cdot \nabla v\right)\right|_{K}=\left.\left(v+q^{r b} \cdot \nabla v\right)\right|_{K} \text { on each } K \in \mathcal{T}_{h} .
$$

Define

$$
\Phi_{n c, h, \ell}^{\epsilon, r b}=J^{\epsilon, r b} \Phi_{\ell}^{h}, \quad \ell=1, \cdots, N_{h} .
$$

Then the reduced basis multiscale finite element space, defined in the spirit of [14], is

$$
V_{n c, h}^{\epsilon, r b}=\operatorname{span}\left\{\Phi_{n c, h, \ell}^{\epsilon, r b}\right\}_{\ell=1, \cdots, N_{h}} .
$$

The Galerkin RB-MsFEM formulation of the problem using $V_{n c, h}^{\epsilon, r b}$ is: Seek $u_{h}^{\epsilon, r b} \in V_{n c, h}^{\epsilon, r b}$, such that

$$
a\left(u_{h}^{\epsilon, r b}, v_{h}^{\epsilon}\right)=f\left(v_{h}^{\epsilon}\right), \quad \forall v_{h}^{\epsilon} \in V_{n c, h}^{\epsilon, r b} .
$$

We can prove the following convergence theorem in the spirit of (4.1):

THEOREM 4.2. Let $u^{\epsilon}$ be the solution of 2.2) and $u_{h}^{\epsilon, r b}$ be the RB-MsFEM solution of (4.27). There exists a positive constants $C$ independent of $\epsilon$ and $h$, such that

$$
\left\|\nabla\left(u^{\epsilon}-u_{h}^{\epsilon, r b}\right)\right\|_{0} \leq C\left(h^{k}+\sqrt{\frac{\epsilon}{h}}+\left(\frac{h^{\prime}}{\epsilon}\right)^{k^{\prime}}+\sqrt{N_{h}} \delta\right) .
$$

An over-sampling technique can also be applied. Rather than building a reduced basis on $K$, we can build the reduced basis on a larger domain $S$ with $K \subset S$, and satisfying Assumption 2.1 of [17]. We likewise assume $S$ is of a uniform size for all elements. After having computed the reduced basis function on $S$ with tolerance $\delta$, we can restrict it to $K$ to recover the local oscillating test function on $K$. Let us denote this function on $K$ by $w_{o s}^{\epsilon, r b}$.

We can now define the oversampling RB-based conforming multiscale finite element basis in the spirit of [4,

$$
\Phi_{c, \ell, o s}^{\epsilon, h, r b}=\Phi_{\ell}^{h}\left(w_{o s}^{\epsilon, r b}(x)\right)
$$

The corresponding RB multiscale finite element space is defined as

$$
V_{c, h, o s}^{\epsilon, r b}=\operatorname{span}\left\{\Phi_{c, \ell, o s}^{\epsilon, r b}\right\}_{\ell=1, \cdots, N_{h}} .
$$

The Petrov-Galerkin RB-MsFEM of the problem is: Seek $u_{h, o s}^{\epsilon, r b} \in V_{c, h, o s}^{\epsilon, r b}$, such that

$$
a\left(u_{h, o s}^{\epsilon, r b}, v_{h}\right)=f\left(v_{h}\right), \quad \forall v_{h} \in V_{h} .
$$

Since $K \subset S$, the following error estimate holds:

$$
\left\|\nabla\left(w^{\epsilon, h}-w^{\epsilon, r b}\right)\right\|_{0, K} \leq\left\|\nabla\left(w^{\epsilon, h}-w^{\epsilon, r b}\right)\right\|_{0, S} \leq \delta, \quad \forall K \in \mathcal{T}_{h} .
$$


TheOrem 4.3. Assume that the coefficient tensor is periodic, i.e., $A^{\epsilon}=a\left(\frac{x}{\epsilon}\right)$, and assume that the size of the over-sampling domain is large enough to ensure that Assumption 2.1 of [17] holds. Then the discrete problem (4.30) is well-posed.

Let $u^{\epsilon}$ be the exact solution of the original problem (2.2) and $u_{h}^{\epsilon}$ be the solution of (3.8). Assume that $u^{*} \in W^{k+1, \infty}(\Omega)$ and $\chi_{i} \in W^{1, \infty}(Y)$. Then exists a constant $C$ independent of $\epsilon$ and $h$ such that

$$
\left\|\nabla\left(u^{\epsilon}-u_{h, o s}^{\epsilon, r b}\right)\right\|_{0} \leq C\left(h^{k}+\sqrt{\epsilon}+\left(\frac{h^{\prime}}{\epsilon}\right)^{k^{\prime}}+\sqrt{N_{h}} \delta\right) .
$$

The above theorem is proved by combining the proof of convergence theorem in [14] and the proof of Theorem 4.2

For a function $v \in H^{1}(\Omega)$, introduce a map $J_{o s}^{\epsilon, r b}$ such that

$$
\left.J_{o s}^{\epsilon, r b} v\right|_{K}=\left.\left(v+\left(w_{o s}^{\epsilon, r b}-x\right) \cdot \nabla v\right)\right|_{K} \text { on each } K \in \mathcal{T}_{h} .
$$

Define the oversampling RB-based conforming multiscale finite element basis in the spirit of [14],

$$
\left.\Phi_{n c, \ell, o s}^{\epsilon, h, r b}\right|_{K}=\left.J_{o s}^{\epsilon, r b} \Phi_{\ell}^{h}\right|_{K}
$$

The corresponding reduced basis multiscale finite element space is defined as

$$
V_{n c, h, o s}^{\epsilon, r b}=\operatorname{span}\left\{\Phi_{n c, \ell, o s}^{\epsilon, r b}\right\}_{\ell=1, \cdots, N_{h}} .
$$

The Petrov-Galerkin RB-MsFEM of the problem is: Seek $u_{h, o s}^{\epsilon, r b} \in V_{n c, h, o s}^{\epsilon, r b}$, such that

$$
a\left(u_{h, o s}^{\epsilon, r b}, v_{h}\right)=f\left(v_{h}\right), \quad \forall v_{h} \in V_{h} .
$$

In the same spirit, we can prove the following convergence theorem

THEOREM 4.4. Assume that the coefficient tensor is periodic, i.e., $A^{\epsilon}=a\left(\frac{x}{\epsilon}\right)$, and assume the size of over-sampling domain is large enough to ensure that Assumption 2.1 of [17] holds. Then the discrete problem (4.35) is well-posed.

Let $u^{\epsilon}$ be the exact solution of the original problem (2.2) and $u_{h}^{\epsilon}$ be the solution of (3.15). Assume that $u^{*} \in W^{k+1, \infty}(\Omega)$ and $\chi_{i} \in W^{1, \infty}(Y)$. Then there exists a constant $C$ independent of $\epsilon$ and $h$ such that

$$
\left\|\nabla\left(u^{\epsilon}-u_{h, o s}^{\epsilon, r b}\right)\right\|_{0} \leq C\left(h^{k}+\sqrt{\epsilon}+\left(\frac{h^{\prime}}{\epsilon}\right)^{k^{\prime}}+\sqrt{N_{h}} \delta\right) .
$$

Note that the restriction of function $\left.w^{\epsilon, r b}\right|_{K}$ needs not to be computed explicitly since we are only interested in the numerical integration on the element $K$. By the affine assumption and the fact that the size of $K$ is uniform, all the required coefficients can be computed in the offline stage. The online assembling of the both sides of (4.35) does not depend on the DOFs on $K$ or $S$.

REMARK 4.5. In principle, instead of choosing the center of the element as parameters and keeping the element size uniform, we can use both the size and the center of the element as parameters. The disadvantage of this approach is that even in the simple periodic case, different sizes of elements will result in completely different local oscillating test functions. In such a case, a reduced basis set with a small number of basis functions is impossible, thus adversely impacting the efficiency of the method.

REMARK 4.6. In this section, we only discussed the reduced basis method for the non-periodic case. For the purely periodic case, the local problem only needs to be solved once, and a reduced 
basis is not needed. For the second special case, $A^{\epsilon}=a(x, x / \epsilon)$, and the entries of the matrix are periodic functions with period $p_{i}$ in the direction of $x_{i}$ of the second variable, $i=1, \cdots, d$. A reduced basis can be built in a similar fashion, with the only difference being that we should take advantage of the periodic boundary conditions for better accuracy during the construction of the basis.

4.3. Numerical experiments. We consider the numerical test with the following multiscale conductivity tensor with diagonal entries are

$$
a_{11}=a_{22}=\left(2+x_{1} \sin \left(x_{1} / \epsilon\right)\right)\left(2+x_{2} \sin \left(x_{2} / \epsilon\right)\right)
$$

the corresponding homogenized tensor is also diagonal can be computed as

$$
a_{11}^{*}=\frac{2}{\frac{1}{2 \pi} \int_{0}^{2 \pi} \frac{1}{2+x_{1} \sin \left(x_{1} / \epsilon\right)} d x_{1}}, \quad a_{22}^{*}=\frac{2}{\frac{1}{2 \pi} \int_{0}^{2 \pi} \frac{1}{2+x_{2} \sin \left(x_{2} / \epsilon\right)} d x_{2}}
$$

where we choose $\epsilon=0.001$ and $u^{*}=(1-x)(1-y) x y$ to be the homogenized solution. The tests are done on the unit square domain $[0,1] \times[0,1]$. We use the $P_{1}$ RB-MsFEM (Galerkin), $h=1 / 50, h^{\prime}=1 / 5000$. Figure 4.1 (left) shows the RB error measured in energy norm (the maximum of error in energy norm over the training set) and a posteriori error estimation through $\mathrm{RB}$ measured in energy norm for the construction of the local oscillating test function $w_{1}, w_{2}$ and exponential convergence is clearly observed. Figure 4.1 (right) shows the convergence of the homogenized solution computed by RB-MsFEM (measured in $L^{2}, H^{1}$ and energy norms), the convergence (measured in $L^{2}$ norm) stops around $10^{-3}$ (corresponds to $5 \mathrm{RB}$ basis functions), indicating that the error of the multiscale approximation $\left(\left\|u^{\epsilon}-u_{h}^{\epsilon, f e}\right\|\right)$ dominates over the error induced by the RB approximation $\left(\left\|u_{h}^{\epsilon, f e}-u_{h}^{\epsilon, r b}\right\|\right)$ and we can control the online computational cost with comparable accuracy by choosing an appropriate RB tolerance.
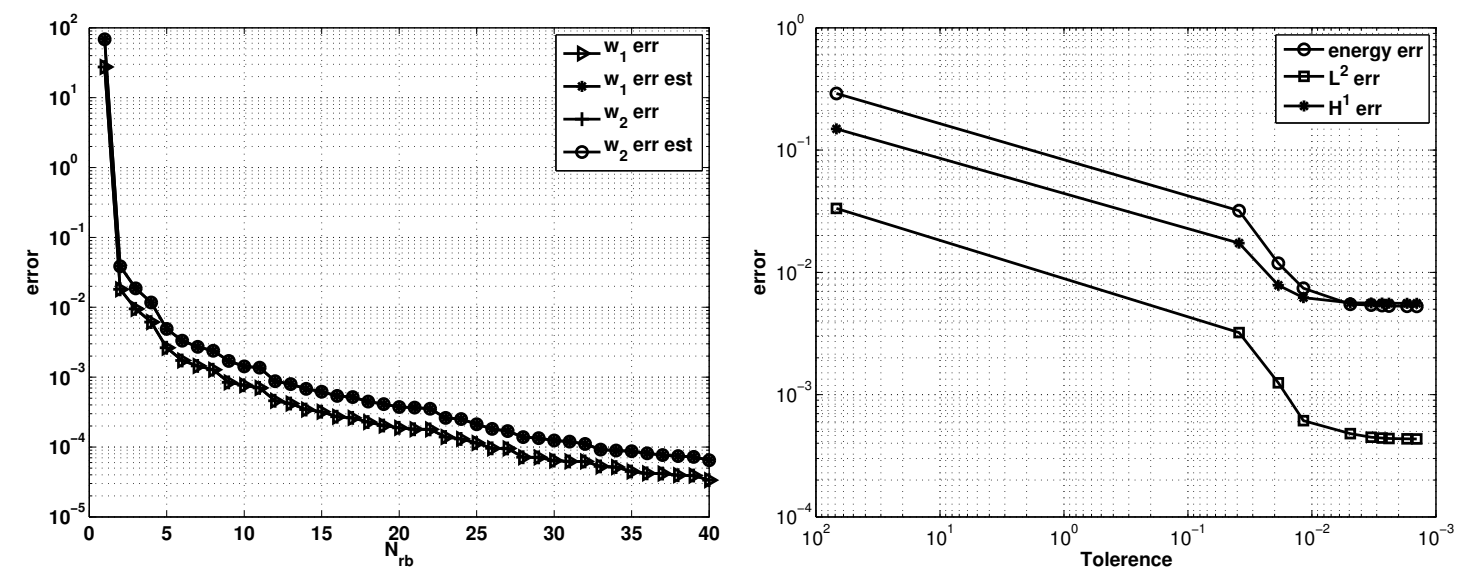

FIGURE 4.1. RB error in energy norm and a posteriori estimation of the RBM during the construction of the set of local oscillating functions $w_{1}, w_{2}$ (left); the error between the exact homogenized solution and the homogenized solution computed by RB-MsFEM and with different $R B$ a posteriori tolerance (right)

5. RB-MsFEM for triangular meshes. The use of triangular meshes is essential for the effective application of finite element methods to handle problems with complex geometry and enable the use of locally adaptive refinement. Hence, it is desirable to develop a fast reduced basis method to recover the local oscillating test functions in the case of triangular elements. In principle, we can use the vertices of the simplex as the parameters. However, as discussed previously, this approach will result in a basis set with a very large number of basis functions and is not recommend. 
We begin by recalling that the central advantage of using oversampling is that the restriction of a local oscillating test function, computed in a larger domain $S$ to a smaller domain $K \subset S$, is always superior to the approach where the local oscillating test function computed on $K$ directly. The reason for this is the reduction of the impact of the artificial boundary condition imposed on the local problem. Exploring this observation, we break the computation of local oscillating test functions on the triangular element $K$ into several steps. The algorithm is given in Algorithm 1 .

1: Build (offline) a reduced basis set for the local oscillating test functions on uniform rectangular domains with element size $h=\left(H_{1}, H_{2}, \cdots, H_{d}\right)$. The reduced basis is parametrized by the center $x_{K}$ of the element.

2: For a given triangular element $K$, associate it with a larger rectangular element $S$ with size $h$.

3: Compute the local oscillating test function $w_{S}$ on $S$ by the reduced basis model

4: Use the restriction of $w_{S}$ on $K$ as the local oscillating test function $w_{K}$ on $K$

Algorithm 1: Algorithm to build local oscillating test function on a triangular element $K$

This algorithm is unique as we have two choices when building the reduced basis method for the uniform rectangular mesh. One is to use a fixed uniform rectangular mesh and assume that each triangular element will belong to one rectangular element. In such a case, we can choose the parameter domain $\mathcal{D}$ to be the collection of the centers of this fixed uniform rectangular mesh and build the reduced basis based on this. The advantage of this is that the parameter domain is discrete and finite. When we seek to restrict the local oscillating test functions to triangles, the process is well defined since we assume that each triangle is associated with a uniform rectangular element. However, a possible disadvantage of this approach is that the boundary of the oversampling domain $S$ may not always be far away enough from the element $K$, thus violating Assumption 2.1 of [17]. In this case, the Petrov-Galerkin formulation is not inf-sup stable, only the standard Galerkin formulation is possible and the method will suffer from the resonance error.

As an alternative, we can build a reduced basis set for all rectangular elements with size $h$ and center $x \in \Omega$. In this case, the parameter domain is continuous and covers the whole computational domain. To compute the local oscillating test functions on a triangular element $K$, we associate the element $K$ with a larger rectangular element $S$ with size $h$ through the center of the element. In this case, we can ensure that $K$ is in the center of $S$ and Assumption 2.1 of [17] can be guaranteed. Hence, a Petrov-Galerkin formulation is stable and the resonance error can be eliminated.

The a priori error estimations are similar to the results for the uniform rectangular mesh case discussed in Sec. 4. For the simplicity of presentation, we omit listing the results.

Unlike the method on the uniform rectangular mesh, the operation counts of the RB-MsFEM on triangular meshes dependent on the local DOFs of the FEM on $S$ since the restriction operation is performed on triangles of different sizes. However, the most costly step, recovering the local oscillating function on $S$, is achieved by the reduced basis method and is RBM therefore independent of the DOFs of the FEM on $S$.

Note that for a general complex domain, it may not possible to extend a triangular element into a larger uniform rectangular element, i.e., this is generally not possible for elements close to the boundary. For such cases, we can compute the local oscillating test function of these element directly by a finite element methods. Since the number of these elements can be expected to comprise a small fraction of the total number of elements in the mesh, this is generally acceptable.

5.1. An inexact restriction. Let us also discuss a few implementation issues. The solution on $S$ is computed on a local finite element mesh $\mathcal{T}(S)$. When restricting the function $w$ on $S$ to $K$, the mesh $\mathcal{T}(S)$ will not generally be aligned with the edges of $K$. If an exact interpolation is used, the process is both complicated and time consuming. 
Consider, as an example, a RB-MsFEM Galerkin formulation and denote $\mathcal{T}^{\prime}(K)$ to be the collections of local finite elements of $\mathcal{T}(S)$ whose center is inside $K$. Rather than computing

$$
\int_{K} A^{\epsilon} \nabla \Phi_{\ell}^{h}\left(w^{\epsilon, r b}\right) \nabla \Phi_{m}^{h}\left(w^{\epsilon, r b}\right) d x, \quad \text { and } \quad \int_{K} f \nabla \Phi_{m}^{h}\left(w^{\epsilon, r b}\right) d x
$$

we compute the approximations:

$$
\sum_{T \in \mathcal{T}^{\prime}(K)} \int_{T} A^{\epsilon} \nabla \Phi_{\ell}^{h}\left(w^{\epsilon, r b}\right) \nabla \Phi_{m}^{h}\left(w^{\epsilon, r b}\right) d x \quad \text { and } \sum_{T \in \mathcal{T}^{\prime}(K)} \int_{T} f \Phi_{m}^{h}\left(w^{\epsilon, r b}\right) d x .
$$

and denote the bilinear and linear forms with the above inexact numerical integration by $a^{*}(u, v)$ and $f^{*}(v)$. Since the size of the local elements is $h^{\prime}$, assumed much smaller than $h$, it's reasonable to assume that the bilinear form $a^{*}(u, v)$ is still coercive. Furthermore, the ratio between the element area on the boundary of $K$ and the area of $K$ is $O\left(h^{\prime} / h\right)$. Using the ideas of Lemma 4.1 and Theorem 4.1 of [26], it is reasonable to assume that

$$
\left\|\nabla\left(u_{h}^{\epsilon, r b}-\tilde{u}_{h}^{\epsilon, r b}\right)\right\|_{0} \leq C \frac{h^{\prime}}{h}
$$

where $\tilde{u}_{h}^{\epsilon, r b}$ is the solution recovered through inexact numerical integration. Since we assume $h>\epsilon$ and the term $\left(\frac{h^{\prime}}{\epsilon}\right)^{k^{\prime}}$ appears in all error estimates, it is safe to use this inexact restriction when a linear approximation is used locally.

5.2. Numerical experiments. We consider the similar numerical test in Section 4.3 on a uniform triangular mesh by the $P_{1}$ RB-MsFEM (Galerkin) with oversampling, $h=1 / 100, h^{\prime}=$ $1 / 5000, h_{o s}=5 h$. Figure 5.1 (left) shows the RB error measured in energy norm and error estimation measured in energy norm for the construction of the local oscillating test function $w_{1}, w_{2}$ and exponential convergence is clearly observed. Figure 5.1 (right) shows the convergence of the homogenized solution computed by RB-MsFEM (measured in $L^{2}, H^{1}$ and energy norms), the convergence (in $L^{2}$ norm) stops around $10^{-3}$ (corresponds to $4 \mathrm{RB}$ basis functions), indicating that the error of the multiscale approximation $\left(\left\|u^{\epsilon}-u_{h}^{\epsilon, f e}\right\|\right)$ dominates over the error induced by the RB approximation $\left(\left\|u_{h}^{\epsilon, f e}-u_{h}^{\epsilon, r b}\right\|\right)$.

Next, we consider the same multiscale tensor coefficient $a\left(\frac{x}{\epsilon}\right)$ with $\epsilon=0.02, f=1$ in an Lshape domain. The nonuniform mesh of coarse elements is illustrated in Figure 5.2 There are 104 elements with $h_{\max }=1 / 4, h_{\max }^{\prime}=h / 100$. Figure 5.2 (right) shows the RB error measured in energy norm and error estimation measured in energy norm for the construction of the local oscillating test function $w_{1}, w_{2}$ and exponential convergence is clearly observed. Figure 5.3 compares the corresponding homogenized solution with $20 \mathrm{RB}$ basis functions and the homogenized solution by solving the homogenized equation through a $P_{1}$ finite element method under the same coarse mesh. Both results agree with each other very well.

6. RB-MsFEM for more general parameter dependent PDEs. In the following, we extend the methods to include more general parameter dependent problems. Consider the elliptic problems of the form

$$
\begin{array}{rllll}
-\nabla \cdot\left(A^{\epsilon}(x ; \boldsymbol{\mu}) \nabla u^{\epsilon}\right) & = & f & \in & \Omega \\
u^{\epsilon} & = & 0 & \text { on } & \partial \Omega .
\end{array}
$$

where $A^{\epsilon}(x ; \boldsymbol{\mu})$ is a symmetric positive definite and bounded coefficient matrix. Here $\boldsymbol{\mu}$ is a collection of parameters in some parameter domain $D$ and $A^{\epsilon}(x ; \boldsymbol{\mu})$ is permitted to vary on a small scale $\epsilon$. The variational formulation of the problem is to seek $u^{\epsilon} \in H_{0}^{1}(\Omega)$ such that

$$
a\left(u^{\epsilon}, v ; \boldsymbol{\mu}\right)=f(v) \quad \forall v \in H_{0}^{1}(\Omega),
$$



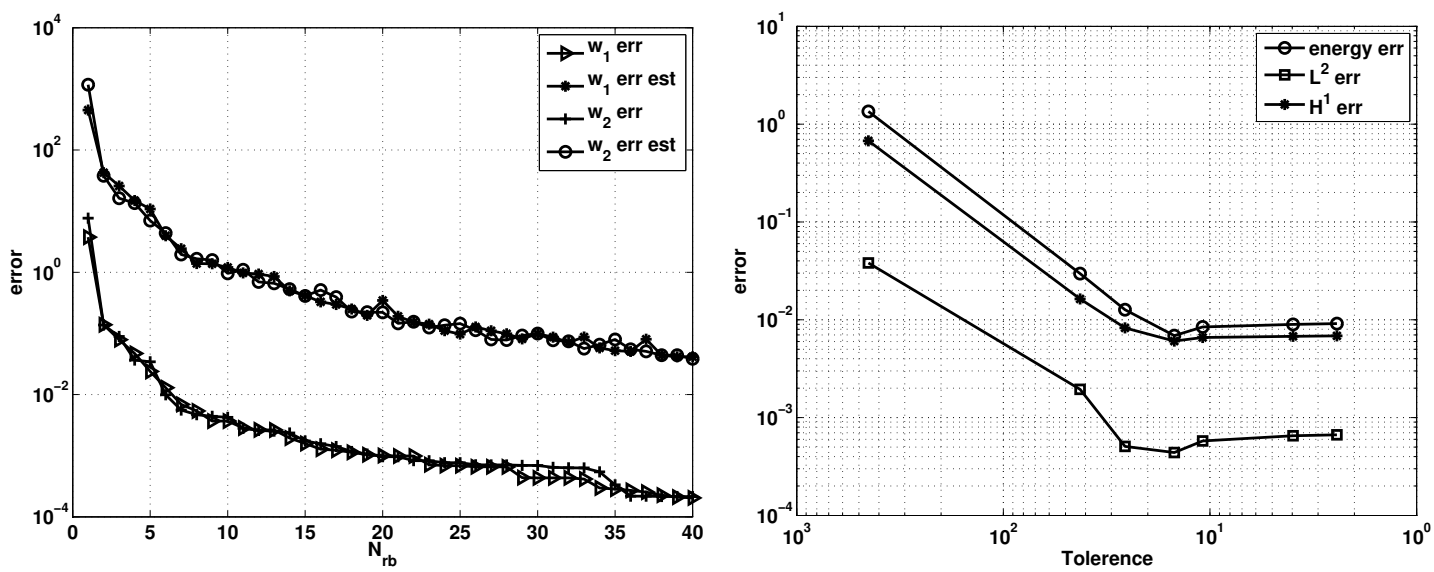

FiguRe 5.1. RB error in energy norm and a posteriori estimation of the RBM during the construction of the set of local oscillating functions $w_{1}, w_{2}$ with a oversampling approach (left); the error between the exact homogenized solution and the homogenized solution computed by RB-MsFEM with different RB a posteriori tolerance (right)
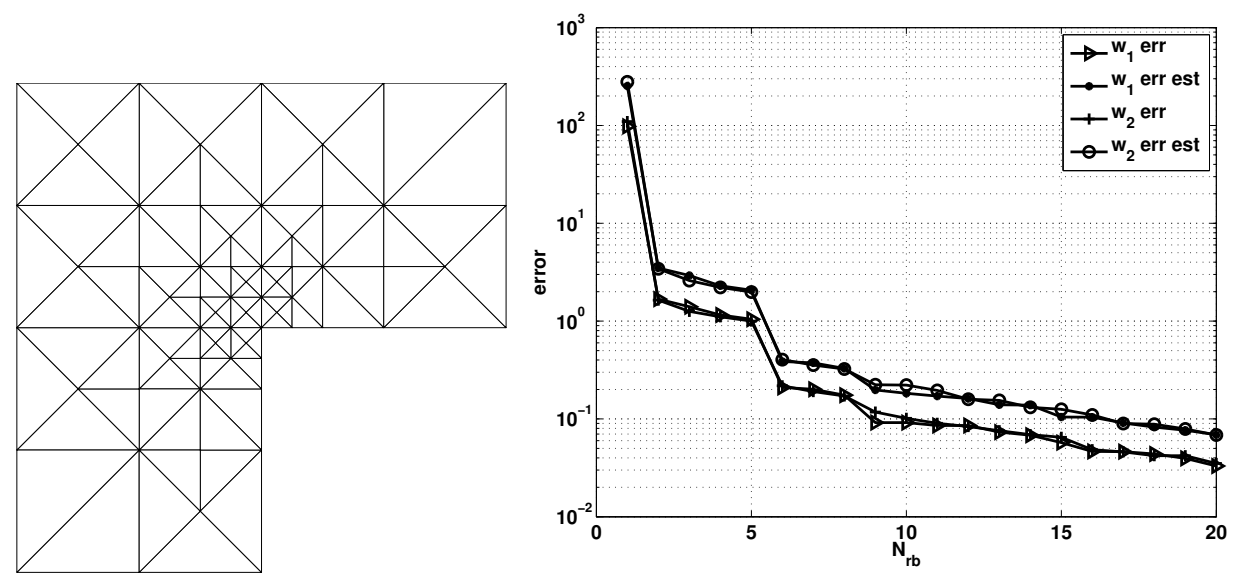

FIgURE 5.2. a nonuniform mesh for the L-shape domain (left); RB error in energy norm and a posteriori estimation of the RBM during the construction of the set of local oscillating functions $w_{1}, w_{2}$ with a oversampling approach (right)

with $a(u, v)=\left(A^{\epsilon}(x ; \boldsymbol{\mu}) \nabla u\right.$ and $\left.\nabla v\right), \quad f(v)=(f, v)$.

This problem can be solved by a standard MsFEM as defined in Sec. 3. Similar to the problem without $\boldsymbol{\mu}$, the local oscillating test function must be solved for each element. Thus, a reduced basis MsFEM is desirable. The RB-MsFEM introduced in Sec. 4 and 5 can be applied in a similar fashion without problems. The main difference is that the parameters are the center of uniform size rectangular element $x_{K}$ and $\boldsymbol{\mu}$. When the problem has high-dimensional parameters, greedy algorithms developed in [12] and ANOVA techniques developed in [13] can be applied to improve the computational efficiency of the reduced basis method for local oscillating test functions.

7. Concluding remarks. In this paper, we developed a new reduced basis multiscale finite element methods (RB-MsFEM) for elliptic problems with highly oscillating coefficients. The method is based on MsFEM with local oscillating test functions(4, 14), and allows for the generalization of RB-MsFEM to high-order methods easily.

For uniform rectangular meshes, the local oscillating test functions are expressed by a reduced 

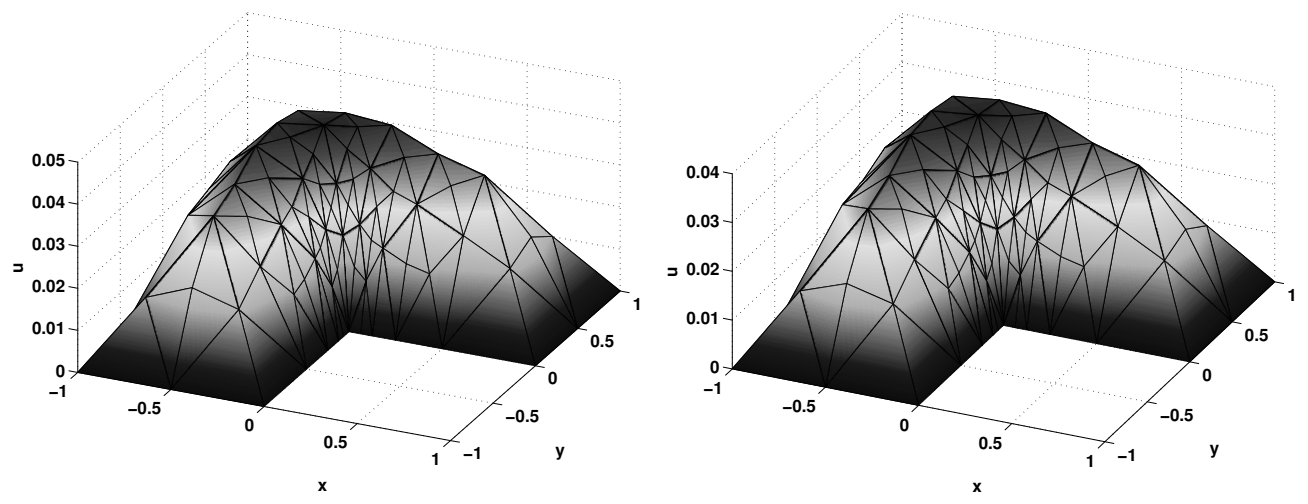

Figure 5.3. Comparison of the homogenized solution computed by RB-MsFEM with 20 RB basis functions (left) and the homogenized solution computed by standard FEM (right)

basis method with element centers as the parameter. The uniform mesh is chosen to make sure that the number of RB functions required is small.

For triangular meshes, we explore the observation that over-sampling oscillating test functions are better approximations of the global harmonic coordinate map than the oscillating test function computed on the non-oversampled domain, we first build the reduced basis set on uniform rectangular elements containing the original triangular elements, then restrict the oscillating test function on triangular elements. This enables the use of a reduced basis multi-scale finite element method for general unstructured grids. The methods generalize to the case of problems whose coefficients also depend on other independent parameters.

The idea of using the restriction of oversampled oscillating test function to smaller elements opens for the possibility for adaptive mesh refinement of in the context of multi-scale finite elements methods and we shall explore such developments in a forthcoming paper.

Acknowledgment. The authors acknowledge partial support by OSD/AFOSR FA9550-09$1-0613$.

\section{REFERENCES}

[1] A. Abdulle And Y. BAI, Reduced basis finite element heterogeneous multiscale method for high-order discretizations of elliptic homogenization problems, J. Comput. Physics, 231, 21, 2012, 7014-7036.

[2] A. Abdulle And Y. Bai,Adaptive reduced basis finite element heterogeneous multiscale method, to appear in Comput. Methods Appl. Mech. Engrg., 2012.

[3] G. Allaire, Shape Optimization by the Homogenization Method, Springer Verlag, New York, 2002

[4] G. Allaire and R. Brizzi, A multiscale finite element method for numerical homogenization, Multiscale Model. Simul. 4, (2005) 790-812.

[5] M. Barrault, N. C. Nguyen, Y. Maday, and A. T. Patera, An "empirical interpolation" method: Application to efficient reduced-basis discretization of partial differential equations, C. R. Acad. Sci. Paris, Ser. I, 339 (2004), pp. 667-672.

[6] A. Bensoussan, J.-L. Lions and G. Papanicolaou, Asymptotic Analysis for Periodic Structures, Volume 5 of Studies in Mathematics and Its Applications, North-Holland, 1978.

[7] S. Boyaval, Reduced-Basis approach for homogenization beyond the periodic setting, Multiscale Model. Simul. 7 (1) (2008) 466-494.

[8] D. Cioranescu and P. Donato, An Introduction to Homogenization, Oxford Lecture Series in Mathematics and Applications 17, Oxford, 1999.

[9] Y. Efendiev And T. Y. Hou, Multiscale Finite Element Methods: Theory and Applications, Springer, 2009.

[10] Y. Efendiev, T. Y. Hou, And X-H. Wu, Convergence of a nonconformal multiscale finite element method, SIAM J. Numer. Anal. 37(2000), 888-910. 
[11] Y. Efendiev, J. Galvisa, And E. Gildin, Local-global multiscale model reduction for flows in high-contrast heterogeneous media, J. Comput. Physics, 231, 24, 2012, 8100-8113.

[12] J. S. Hesthaven, B Stamm, and S. Zhang, Efficient greedy algorithms for high-dimensional parameter spaces with applications to empirical interpolation and reduced basis methods, accepted by M2AN (Math. Model. Numer. Anal.).

[13] J. S. Hesthaven and S. Zhang, On the use of ANOVA expansions in reduced basis methods for highdimensional parametric partial differential equations, Brown University DAM-Scientific Computing Tech Report 23, 2011.

[14] J. S. Hesthaven, S. Zhang, and X. Zhu, High-order multiscale finite element methods for elliptic problems, Brown University DAM-Scientific Computing Tech Report 12, 2012.

[15] T. Y. Hou And X-H. Wu, A multiscale finite element method for elliptic problems in composite materials and porous media, J. Comp. Physics, vol. 134, 1, 1997, 169-189.

[16] T. Y. Hou, X-H. Wu, AND Z. CAI, Convergence of a multiscale finite element method for elliptic problems with rapidly oscillating coefficients, Math. Comp., 68(1999), 913-943.

[17] T. Y. Hou, X-H. Wu, And Y. Zhang, Removing the cell resonance error in the multiscale finite element method via a Petrov-Galerkin formulation, Comm. Math. Sciences, 2(2004), 185-205.

[18] V. Jikov, S. Kozlov, and O. Oleinik, Homogenization of Differential Pperators and Integral Functionals, Springer, Berlin, 1995.

[19] S. Kaulmanna, M. Ohlbergerb, and B. HaAsdonk, A new local reduced basis discontinuous galerkin approach for heterogeneous multiscale problems, Comptes Rendus Mathematique 349 (2011), no. 23-24, $1233-1238$.

[20] Y. Maday, N. C. Nguyen, A. T. Patera, and G. S. H. Pau, A general multipurpose interpolation procedure: the magic points, Commun. Pure Appl. Anal., 8(1):383-404, 2009.

[21] N.C. NGuyen, A multiscale reduced-basis method for parametrized elliptic partial differential equations with multiple scales. J. Comput. Phys., 227 (2007) 9807-9822.

[22] H Owhadi, L. Zhang, Metric-based upscaling, Comm. Pure Appl. Math, 60, 5, 675-723, 2007.

[23] A.T. Patera and G. Rozza, Reduced Basis Approximation and A Posteriori Error Estimation for Parametrized Partial Differential Equations, Version 1.0, Copyright MIT 2006, to appear in (tentative rubric) MIT Pappalardo Graduate Monographs in Mechanical Engineering.

[24] A. Quarteroni, G. Rozza, and A. Manzoni, Certified reduced basis approximation for parametrized partial differential equations and applications, J. Math. Indust., 2011, 1:3.

[25] Reduced Basis at MIT. [http://augustine.mit.edu/methodology.html]

[26] G. Strang and G. J. Fix, An Analysis of the Finite Element Method, Prentice-Hall, 1973.

[27] L. TARTAR, The General Theory of Homogenization. A Personalized Introduction, Lecture Notes of the Unione Matematica Italiana, 7. Springer-Verlag, Berlin; UMI, Bologna (2009). 